\title{
Practical Routing and Channel Assignment Scheme for Mesh Networks with Directional Antennas
}

\author{
Wei Zhou, Xi Chen, Daji Qiao \\ Department of Electrical and Computer Engineering \\ Iowa State University, Ames, IA 50011 \\ \{zhouwei, leon6827, daji\}@iastate.edu
}

\begin{abstract}
Wireless Mesh Network (WMN) has been recognized as one of the promising technologies to provide wireless broadband access. In addition to the multi-radio multi-channel network architecture often seen in WMNs, employment of directional antennas on each mesh node could further improve the system throughput via alleviating the interference between nearby nodes thus allowing more concurrent transmissions in the network. In this paper, we describe a novel algorithm to produce joint decisions on routing and channel assignment with practical implementation considerations for WMNs with directional antennas. In particular, we formulate this joint optimization problem as a Mixed Integer Programming (MIP) problem with all practical considerations modeled explicitly as MIP constraints. For example, our scheme only considers dual-path routes and oneto-one association between access points and gateway nodes, so as to facilitate its deployment with commercial wireless networking devices such as IEEE 802.11 compliant devices. The objective is to maximize the aggregate system throughput and to provide fair and satisfactory services to all access points. Simulation results show that our scheme (i.e., the solution to the MIP problem) fully exploits the multi-radio multi-channel network architecture and directional antennas in WMNs, and is able to achieve the design objectives with significantly reduced implementation complexity.
\end{abstract}

\section{INTRODUCTION}

\section{A. Background and Motivation}

As a new promising technology, Wireless Mesh Network (WMN) is playing an increasingly important role in the next generation of wireless broadband access. A typical WMN consists of a collection of stationary wireless mesh nodes including Mesh Routers (MRs), Access Points (APs) and Gateway Nodes (GNs). Mesh nodes form a communication backbone which relays traffic between APs and GNs through one or multiple paths. The primary goal of WMN is to provide end users (static or mobile) high-speed Internet access via APs with the less expensive and easier-to-deployment wireless infrastructure than the wired counterparts [1].

One of the key design considerations of WMN is to maximize the system aggregate throughput and to provide fair and satisfactory services to APs. In order to achieve these goals, the critical interference issue needs to be addressed. It has been well known that the interference among transmissions operating on the same frequency channel may be alleviated by using multiple radios on each mesh node and by assigning

The research reported in this paper was supported in part by the Information Infrastructure Institute (iCube) of Iowa State University, and the NSF under Grants No. CNS 0520102 and No. CNS 0716744. different channels to each radio, thus enabling more concurrent transmissions. Although such a multi-radio multi-channel network architecture allows more simultaneous transmissions in the network, it cannot eliminate the interference completely due to limited number of available non-overlapping channels and broadcast nature of the wireless medium. For example, the IEEE 802.11a physical layer (PHY) [2] offers 12 nonoverlapping channels while there are only 3 in the IEEE 802.11b PHY [3]. Any radio within the interference range of a radio with omnidirectional antenna (which is approximately a disk centered at the radio) will be affected if they operate on the same frequency channel.

Using directional antennas in $\mathrm{WMN}$ has been recognized as an attractive solution to exploit spatial reuse as well as frequency diversity, hence to further ameliorate the interference problem. With the same number of available non-overlapping channels, networks using directional antennas typically allow more parallel transmissions than those using conventional omnidirectional antennas.

\section{B. Contributions}

In this paper, we consider a multi-radio multi-channel wireless mesh network with each radio equipped with a directional antenna, and study the problems of routing and channel assignment jointly. The key contributions are:

- We study this joint problem with practical implementation considerations. In order to facilitate the deployment of our scheme, we only consider dual-path routes and oneto-one association between APs and GNs, which reduces the implementation complexity significantly.

- We formulate this joint optimization problem as a Mixed Integer Programming (MIP) problem. The MIP objective function is to find a scheme which strikes the balance between maximizing the aggregate system throughput and providing fair services to the APs. All practical considerations are modeled explicitly as MIP constraints.

- We evaluate our scheme (i.e., the solution to the MIP problem) by extensive QualNet [4] simulations. Results clearly show that our scheme is able to meet the design goals via making efficient use of available radios and nonoverlapping frequency channels in the network.

\section{Related Work}

We now review the previous work related to network performance optimization in WMNs. In [5], the authors presented a 
maximum throughput and fair bandwidth allocation algorithm for multi-radio multi-channel WMNs, where channel assignment was predefined [6] and considered independently. The authors of [7] proposed a centralized channel assignment and routing scheme based on heuristic route discovery and traffic load estimation. In [8], a dual-path routing selection metric was proposed to consider both link quality and interference. However, each node performs route selection independently, which leads to sub-optimal solutions. In [9], tree-based network structure and routing protocol were proposed. Unfortunately, single-path routing in such tree structure cannot fully exploit the parallel transmissions offered by multi-path routing in multi-radio multi-channel WMNs. The authors of [10] formulated the joint routing and channel assignment problem as a Linear Programming (LP) to optimize the overall network throughput subject to fairness constraints on clients. The algorithm begins by solving a network max-flow LP problem, which may violate certain practical constraints, followed by a set of post processing in order to round the LP results to a feasible solution. Moreover, channel assignment is performed after routing has been determined, which may not be optimal. A similar approach was proposed in [11]. Time synchronization is required in both algorithms.

One of the potential issues of the above schemes is the significant implementation complexity: (i) an AP may be associated with multiple GNs which requires all GNs to cooperate for flow scheduling; (ii) a flow may travel through many paths which increases the complexity of the routing protocol; and (iii) requirement of time synchronization among mesh nodes renders such algorithms very difficult to implement, particularly with commercial IEEE 802.11 devices. The above observations motivate us to design an efficient, and more importantly simple and easy-to-implement, routing and channel assignment scheme for WMNs with directional antennas.

\section{Organization}

The rest of this paper is organized as follows. System models are given in Section II and the problem formulation is presented in Section III. In Section IV, we describe the construction of auxiliary graph and the MIP formulation in detail. Through extensive simulation, the performance of our proposed scheme is evaluated in Section $\mathrm{V}$ and the paper concludes in Section VI.

\section{SySTEM Models}

\section{A. Network Architecture}

We consider a multi-radio multi-channel Wireless Mesh Network (WMN) consisting of stationary mesh nodes at known locations. Each mesh node is equipped with multiple IEEE 802.11 radios using directional antennas. As shown in Fig. 1, there are three types of mesh nodes:

- Access Point (AP) - mesh node which has Access Point functionality and provides last-hop access services to end users within its coverage area;

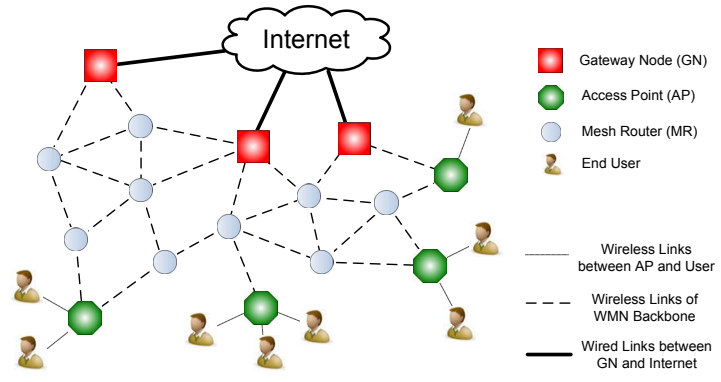

Fig. 1. An example wireless mesh network

- Gateway Node (GN) - mesh node which connects to the Internet through the wired link with unlimited bandwidth;

- Mesh Router (MR) - mesh node which relays traffic between APs and GNs.

Mesh nodes are connected through wireless links to form the communication backbone of WMN. Traffic between end users and the Internet will be relayed over one or multiple paths through the WMN. In practice, quantity and locations of mesh nodes vary with the area of deployment, demand of services, and availability of resources.

We assume that all radios operate in half-duplex mode, i.e., a radio can only transmit or receive at any time. We say that there exists a link between two nodes if (i) they have radios pointing to each other and operating on the same frequency channel, and (ii) they are within the transmission range of each other. Two links are called adjacent if they have a node in common. Link capacity is defined as the highest possible data transmission rate over the link. Moreover, we assume symmetric links in the network; hence without loss of generality, we only consider traffics from APs to GNs in this work.

Formally, the WMN under consideration can be modeled as an undirected graph $G=(V, E)$, where each node $v \in V$ is equipped with $d(v)$ radios with directional antennas. We use $\Delta(G)$ to denote the maximum degree of the nodes in $G$, i.e., $\Delta(G)=\max \{d(v) \mid v \in V\}$. Moreover, we use $e(u, v) \in E$ to represent the bi-directional link between nodes $u$ and $v$ with capacity $C_{e} . K$ stands for the total number of available nonoverlapping frequency channels in the network.

\section{B. Directional Antenna and Interference Model}

With an omnidirectional antenna, the interference range of a radio can be approximately modeled as a disk centered at the radio, and all nodes inside the disk are affected if their radios operate on the same frequency channel. How to improve the throughput by separating transmissions in the frequency domain has been well studied. However, the extent of improvement is strictly limited by the number of available non-overlapping channels. By contrast, usage of directional antennas offers spatial separation between contending transmissions hence may further improve the network performance. In this paper, we consider mesh nodes equipped with multiple radios and each radio uses a practical low-cost switch-beam directional antenna with a fixed transmitting/receiving direction. 
Since the transmit power of a directional antenna focuses in one direction and forms a cone-shape pattern, one can expect better spatial usage compared to an omnidirectional antenna. For example, as shown in Fig. 2(a), there is no interference between transmissions $\mathrm{M} 2 \rightarrow \mathrm{T}$ and $\mathrm{S} \rightarrow \mathrm{M} 3$; hence they may proceed at the same time. However, if node M2 uses an omnidirectional antenna with interference range $r$, these two transmissions can not take place at the same time because M3 is within M2's interference range and their radios work on the same channel.

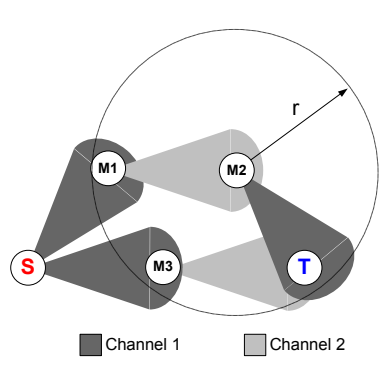

(a) Directional antenna: spatial reuse

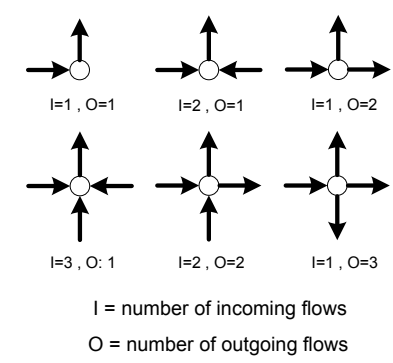

(b) Example interference patterns in a network with $\Delta(G)=4$

Fig. 2. Directional antenna and interference model

Unfortunately, interference cannot be eliminated completely even with directional antennas. Interference occurs if (i) a node is located within the cone-shape area of transmitter's radio, and (ii) it has radio(s) operating on the same frequency channel as the transmitter's radio. We assume that the transmit power of each directional antenna is set properly so that they are able to communicate with one-hop neighbors while causing minimal interference to others. Thus, we only consider the interference among adjacent links. Moreover, in order to characterize the severity level of the interference on the common node of adjacent links, we introduce a heuristic interference factor, denoted by $\phi$. Fig. 2(b) illustrates several example interference patterns in a network with maximum degree of 4 . In general, a larger $\phi$ is assigned to an interference pattern with more incoming/outgoing flows. In practice, $\phi$ values are determined by experimental measurements at the initial stage of the WMN deployment.

\section{Problem Statement}

\section{A. Design Objectives}

The goal of this work is to design a multi-objective algorithm to produce joint decisions on routing and channel assignment in multi-radio multi-channel WMNs. The primary objective is to maximize the aggregate system throughput, which measures the efficiency of network resource utilization. However, simply maximizing the aggregate system throughput may lead to starvation of certain APs. To address this fairness issue, the second objective is to maximize the minimum bandwidth allocation among APs. Further, we add the third objective which is to minimize the total hop count if the first two objectives have been achieved. In other words, if there exist multiple solutions that satisfy the first two objectives, our algorithm picks the one with minimum hop count in order to minimize the network resource utilization.

Most of previous work have proposed to perform channel assignment after the routing decision has been made [1], [5], which may not be optimal. In contrast, our algorithm is designed to consider routing and channel assignment together by solving a joint optimization problem, so as to yield better network performance.

\section{B. Dual-Path Routing and One-to-One AP-GN Association}

Routing in a graph with link capacity limitation is usually formulated and solved as a max-flow problem, which finds a maximum-rate flow in a single-source single-sink network. The max-flow method may also be used in a multi-source multi-sink network by introducing a hyper source (which connects to all sources) and a hyper sink (which connects to all sinks). It has been shown that multi-path routing can significantly improve the end-to-end throughput in multi-radio multi-channel wireless networks [1], [8], [9]. However, from practical implementation point of view, such approaches have several inherent limitations.

First, in the max-flow problem, each flow is allowed to travel through unlimited number of paths which makes the corresponding routing protocol very complicated. On the other hand, previous work [8], [12], [13] have shown that dual-path routing protocol efficiently exploits the feature of multi-path routing with much lower implementation complexity. For this reason, we add a feasibility constraint in our joint optimization problem: route selection is limited to dual-path routes. Simulation results in Section V demonstrate the effectiveness of dual-path routing in multi-radio multi-channel WMNs.

Second, in the max-flow problem, there is no restriction on the AP-GN association. One AP may be associated with multiple GNs. From practical implementation point of view, this may be technically challenging because multiple GNs have to cooperate with each other in order to service one AP. Hence, we add another feasibility constraint in our joint optimization problem: each AP can only associate with one GN. This further reduces the complexity of the routing protocol generated by our algorithm.

\section{Joint Routing And CHAnnel Assignment}

In this section, we describe the details of our proposed joint routing and channel assignment scheme. It consists of two steps. First, given a network graph $G$, we construct an auxiliary graph $G^{\prime}$ to model the following constraints explicitly:

- General network constraints including the general flow constraint, number of radios on each node and total number of available non-overlapping channels, which will be formulated in Section IV-B;

- Feasibility constraints for implementation considerations: dual-path routing and one-to-one AP-GN Association, which were discussed in Section III-B and will be formulated in Section IV-B. 
Second, we formulate the problem of making joint decisions on routing and channel assignment as a Mixed Integer Programming (MIP) problem. The output of the MIP includes (i) the AP-GN associations, (ii) the flow rate of each AP-GN pair on each link, which determines the routes and bandwidth allocated to each AP, and (iii) the channel assigned to each link.

\section{A. Constructing the Auxiliary Graph}

Given a network graph $G=(V, E)$, we construct its auxiliary graph $G^{\prime}=\left(V^{\prime}, E^{\prime}\right)$ in three steps:

- The sets of Access Points (APs), Gateway Nodes (GNs), and Mesh Routers (MRs) in a WMN are denoted by $S=\left\{s_{1}, s_{2} \ldots, s_{|S|}\right\}, T=\left\{t_{1}, t_{2} \ldots, t_{|T|}\right\}$, and $M=$ $\left\{m_{1}, m_{2} \ldots, m_{|M|}\right\}$, respectively. For each MR $m \in M$, we create $|S|+|T|$ virtual nodes in $G^{\prime}$ corresponding to $m$, namely $m^{s_{1}}, m^{s_{2}}, \ldots, m^{s_{|S|}}$ and $m^{t_{1}}, m^{t_{2}}, \ldots, m^{t_{|T|}}$, and denote this set of virtual notes as $M^{\prime}$. All flows coming into $m^{s_{i}}$ are from AP $s_{i}$ and all flows going out from $m^{t_{j}}$ are destined for GN $t_{j}$. Similarly, each AP $s \in S$ is mapped to $|T|$ virtual nodes in $G^{\prime}$ and each GN $t \in T$ is mapped to $|S|$ virtual nodes in $G^{\prime}$.

- Second, for each MR $m \in M,|S| \times|T|$ intra-node links with infinite link capacity $(\infty)$ are created in $G^{\prime}$ which allow traffic to switch between different radios within node $m$, as shown in Fig. 3(b). Note that all intra-node links are omitted in the example $G^{\prime}$ shown in Fig. 3(c) for clarity. Moreover, for each link $e \in E$ between MRs, we create $|S| \times|T|$ virtual links in $G^{\prime}$, each with capacity $C_{e}$. For each link connected to an $\mathrm{AP}$ or a $\mathrm{GN}$ in $G,|T|$ or $|S|$ virtual links are created in $G^{\prime}$.

- Third, since both $G$ and $G^{\prime}$ are undirected graphs, the flow on link $e^{\prime} \in E^{\prime}$, denoted by $f_{e^{\prime}}$, is bounded by $\left[-C_{e}, C_{e}\right]$, meaning that a flow can travel in either direction on a link depending on the algorithm. Note that if multiple flows (from different APs) share a common link, all the flows should follow the same direction on the link.

Fig. 3 shows an example of the above procedure. The original network graph $G$ and its auxiliary graph $G^{\prime}$ are shown in Figs. 3(a) and (c), respectively. For instance, a flow in $G$ $s_{1} \rightarrow m_{1} \rightarrow m_{2} \rightarrow t_{1}$ is mapped to $s_{1}^{t_{1}} \rightarrow m_{1}^{s_{1}} \rightarrow m_{1}^{t_{1}} \rightarrow$ $m_{2}^{s_{1}} \rightarrow m_{2}^{t_{1}} \rightarrow t_{1}^{s_{1}}$ in $G^{\prime}$, which enables us to model the feasibility constraints explicitly and conveniently.

\section{B. MIP Formulation}

We formulate the problem of making joint decisions on routing and channel assignment as a Mixed Integer Programming (MIP) problem, which is shown in Fig. $4 .{ }^{1}$ Other than the notations defined in Section II, Table I summarizes the additional notations used in the MIP formulation.

Our objective is to maximize the aggregate system throughput, to balance the bandwidth allocation among APs, and to

\footnotetext{
${ }^{1}$ The MIP formulation is presented using notations in $G$ for conceptual clarity only. In order to formulate the problem as a network flow model, all constraints and the objective function are transformed into $G^{\prime}$ notations when solving the MIP.
}

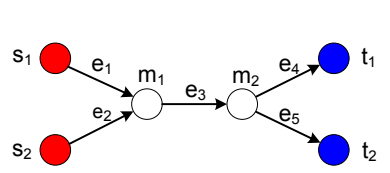

(a) Network Graph G

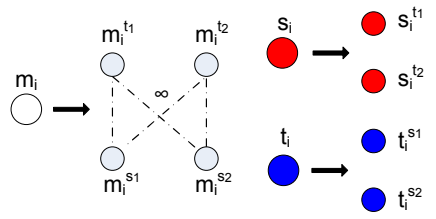

(b) Transformation

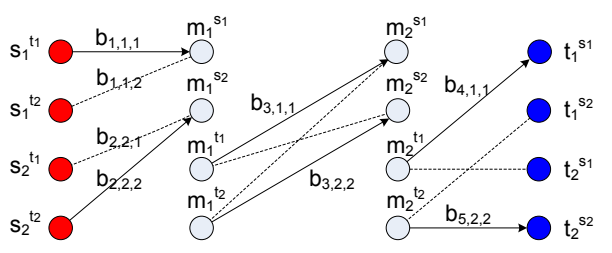

(c) Auxiliary Graph G'

Fig. 3. An example network graph $\mathrm{G}$ and its auxiliary graph $\mathrm{G}$ '

TABLE I

AdDitionAl NOTATIONS USED IN THE MIP FORMULATION

\begin{tabular}{|l||l|}
\hline$F^{s t}$ & A flow from AP $s$ to GN $t$ \\
\hline$f_{u v}^{s t}$ & Rate of flow $F^{s t}$ on link $(u, v)$ \\
\hline$f^{s \rightarrow}$ & Total bandwidth allocated to AP $s$ \\
\hline$H_{F} s t$ & Total number of hops of flow $F^{s t}$ \\
\hline$N(v)$ & Set of neighbor nodes to $v$ \\
\hline$I$ & Network-level interference factor \\
\hline
\end{tabular}

minimize the total hop count if the previous two objectives have been achieved. Correspondingly, as shown in Fig. 4, three parameters, $\alpha, \beta$ and $\gamma$, are introduced to the MIP objective function to weigh the minimum bandwidth allocation among APs, the total hop count, and the interference level, respectively. These parameters can be adjusted by the network designer to reflect different design considerations.

We now explain the meanings of eight constraints in the MIP formulation.

- [C1] is a general flow constraint for all MRs, which ensures that the sum of incoming flows equals the sum of outgoing flows at each MR.

- [C2] states that the total bandwidth allocated to an AP $s$ equals the sum of its outgoing flows.

- [C3] makes sure that the sum of outgoing flows from an AP $s$ equals the sum of incoming flows to its associated GN $t$. Note that the AP-GN associations are output by the MIP. By contrast, in the max-flow problem, the solution only guarantees that the sum of outgoing flows from all APs equals the sum of incoming flows to all GNs.

- [C4] ensures that there is no link capacity violation, meaning that the sum of all flow rates on a link does not exceed the capacity of this link.

Before proceeding to explanations for constraints [C5] to [C8], we first describe two integer variables introduced in the MIP formulation for modeling the feasibility constraints.

- For each link $e^{\prime} \in E^{\prime}$ corresponding to $e_{k} \in E$, we assign a binary variable $b_{k, i, j}$, which equals 1 if a flow from AP $s_{i}$ to $\mathrm{GN} t_{j}$ goes through link $e_{k}$ or 0 otherwise. For example, in Fig. 3(c), $b_{3,1,1}$ is set to 1 if there is a flow 


\begin{tabular}{|lll|}
\hline maximize & $\sum_{s \in S} f^{s \rightarrow}-\gamma \cdot I+\alpha \cdot \min _{s \in S} f^{s \rightarrow}-\beta \cdot \sum_{F} H_{F}$ \\
subject to & \\
[C1] $\sum_{u \neq v} f_{u v}^{s t}=\sum_{v \neq u} f_{v u}^{s t}$, & $\forall v \in M$ \\
[C2] $f^{s \rightarrow}=\sum_{v \in N(s)} f_{s v}^{s t}$, & $\forall s \in S$ \\
[C3] $\quad \sum_{v \in N(s)} f_{s v}^{s t}=\sum_{w \in N(t)} f_{w t}^{s t}$, & $\forall s \in S, v, w \in M$ \\
[C4] $-C_{e} \leq \sum_{s \in S} f_{e}^{s t} \leq C_{e}$, & $\forall e \in E$ \\
[C5] $\quad H_{F^{s} t_{j} t_{j}}=\sum_{e_{k}} b_{k, i, j}$, & $\forall e_{k} \in F^{s_{i} t_{j}}$ \\
[C6] $I=\sum_{v} \phi_{v}$, & $\forall v \in V$ \\
[C7] $\sum_{t_{j} \in T} b_{k, i, j}=1$, & $\forall e_{k} \in E, s_{i} \in S$ \\
[C8] $p_{s, t} \leqslant 2$, & $\forall s \in S$
\end{tabular}

Fig. 4. The formulated MIP problem

from $\mathrm{AP} s_{1}$ to GN $t_{1}$ going through link $e_{3}$ in $G$.

- In order to limit the number of paths for each flow, we associate an integer variable $p_{i, j}$ to a flow from AP $s_{i}$ to GN $t_{j}$. Specifically, $p_{i, j}$ is initially set to 1 and it increments by 1 each time flow $F^{s_{i} t_{j}}$ splits into two flows at AP $s_{i}$ or an MR.

Now we resume to explain constraints [C5] to [C8].

- [C5] represents the total number of hops of flow $F^{s t}$.

- [C6] is a heuristic measurement of the total interference level in the network. $I$ is the sum of interference factors $\phi$ of all nodes, where $\phi_{v}$ is calculated based on the routing and channel assignment on node $v$, as described in Section II.B.

- [C7] limits each AP to associate with only one GN.

- [C8] characterizes the dual-path routing limitation on all flows, which ensures that a flow can not split into more than two paths towards the GN.

The solution to this MIP problem (i) maximizes the aggregate system throughput by selecting the best dual-path routes and minimizing the interference, (ii) maximizes the minimal bandwidth allocation among all APs, and (iii) minimizes the total hop count, while satisfying all the afore-described constraints. Although the MIP problem is known to be NP-hard, our problem can be solved efficiently in a reasonable time using the CPLEX solver [14], thanks to the branch-and-cut technique as well as our application of model optimization and two feasibility constraints, which shrinks the search space of the MIP significantly. This enables our algorithm to dynamically adapt to network condition changes in a timely manner.

\section{Performance Evaluation}

In this section, we evaluate the effectiveness of the proposed scheme using the QualNet simulator [4].

\section{A. Simulation Setup}

In the network we simulated, each mesh node is equipped with multiple radios with directional antennas. IEEE 802.11a MAC and PHY are adopted. We assume that link capacity is determined by the link distance, and each link may transmit at one of the eight available rates: 6, 9, 12, 18, 24, 36, 48, or $54 \mathrm{Mbps}$. Moreover, since the beam width of commonly available directional antennas ranges from $30^{\circ}$ to $60^{\circ}$, we limit the number of radios on each node to 4 to minimize the backlobe and sidelobe effects of directional antennas.

We compare four routing protocols in the simulation: SinglePath; Dual-Path; Triple-Path; and Unlimited-Path which has no restriction on the number of paths from an AP to its associated GN. All routing protocols are generated by the MIP with constraint [C8] adjusted accordingly. We vary the number of available non-overlapping frequency channels $(K)$ from 1 to 4 to study its effect on the network performance. Two types of networks are simulated: Grid Topology and Random Topology.

The parameters in the MIP objective function are set as follows. Recall that $\alpha$ is the weight for fair bandwidth allocation in the objective function. A larger $\alpha$ favors the AP with the minimum allocated bandwidth but may affect the aggregate system throughput. Therefore, we set $\alpha=1$ since the aggregate system throughput is our primary objective. On the other hand, since the total hop count minimization has the lowest priority in the objective function, we set its weight $\beta=1 / L$ (where $L$ is the total number of links in $G$ ), which ensures that the total hop count will be minimized only if the other two objectives have been met. Moreover, since interference plays a critical role in system performance, we set $\gamma=100$ to penalize the interference on adjacent links. $\phi$ values for different interference patterns are determined via simulation.

We evaluate the performance of the simulated routing protocols using two metrics: (i) the aggregate system throughput, which is the total bandwidth allocated to all APs, and (ii) Jain's fairness index [15] of the bandwidth allocations among APs, calculated by

$$
J=\frac{\left(\sum x_{i}\right)^{2}}{n \cdot \sum x_{i}^{2}},
$$

where $x_{i}$ represents the bandwidth allocated to AP $i$ and $n$ is the number of APs. Jain's fairness index lies in $(0,1]$ and $J=1$ corresponds to the best-case scenario when bandwidth is evenly allocated among APs.

\section{B. Grid Topology}

We first study a grid-topology network. As shown in Fig. 5(a), 49 mesh nodes are uniformly deployed in a $1500 \times$ 1500 square meter area and form a $7 \times 7$ grid topology. The distance between neighbor nodes is 250 meters, which corresponds to link capacity of $24 \mathrm{Mbps}$ according to the IEEE 802.11a propagation model in QualNet. We generate 10 scenarios with different AP and GN locations. In each scenario, we randomly selected three nodes as APs and three nodes as GNs. An example scenario is shown in Fig. 5(a). Simulation 


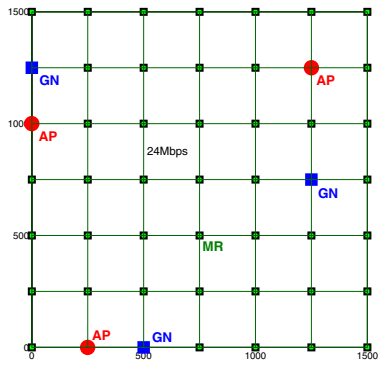

(a) Red circle represents AP, blue square represents GN, and other nodes are MR

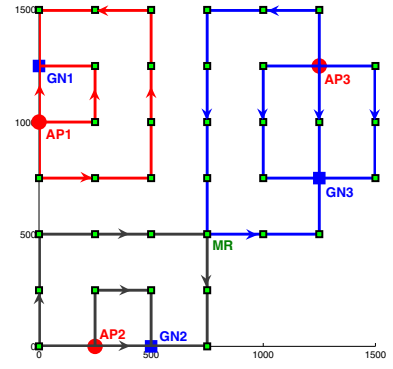

(b) Routing paths in Scenario \#7
Fig. 5. The grid-topology network with 49 nodes

results are shown in Fig. 6 where each point is averaged over 10 scenarios.

We have two observations from Fig. 6. First, the system throughput increases with the number of available nonoverlapping channels $(K)$. The prominent throughput increment is observed when $K$ increases from 1 to 2 . However, one can see that the performance improvement is almost negligible when $K \geqslant 3$. This suggests that we can obtain considerable throughput improvement in multi-radio network with only a small number of non-overlapping channels.

Our second observation is that the proposed dual-path routing protocol can efficiently exploit the multi-radio multichannel architecture of the network and achieve significant throughput improvement compared with the single-path routing protocol. Moreover, as shown in Fig. 6, the marginal gain obtained by triple-path and unlimited-path routing over dualpath routing are very limited in most scenarios. According to our analysis, in grid-topology networks, triple-path and unlimited-path routing show clear advantage only if (i) an AP

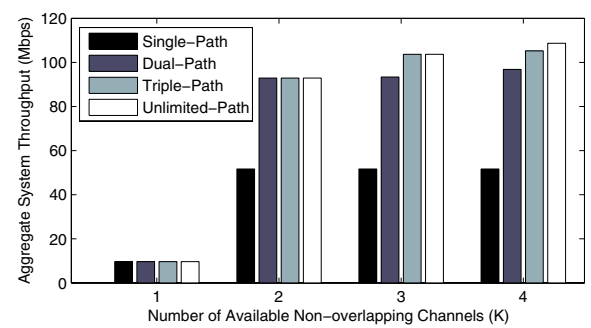

Fig. 6. Comparison of aggregate system throughput in grid-topology networks

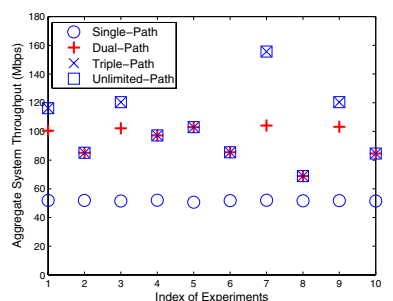

(a) 3 non-overlapping channels

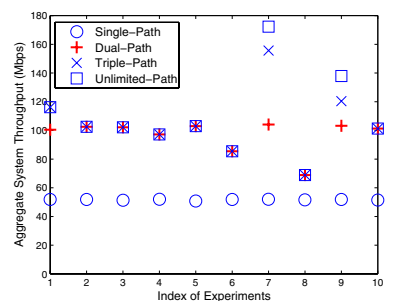

(b) 4 non-overlapping channels
Fig. 7. Results of 10 simulated scenarios in the grid-topology network

can find more than three disjoint paths to its associated GN, and (ii) there are at least three available non-overlapping channels.

In order to study the system performance in detail, we plot the simulation results of all 10 scenarios in Fig. 7. The cases of $K=1,2$ are omitted since the performance difference are less significant due to severe interference. Compared with dual-path routing, unlimited-path routing improves the system throughput by $65.5 \%$ at most, which occurs in Scenario \#7 as shown in Fig. 5(b). In this scenario, both AP1 and AP2 have three disjoint paths to their associated GNs (GN1 and GN2 respectively), and AP3 has four disjoint paths to GN3. Therefore, all the transmissions shown in Fig. 5(b) can take place simultaneously when $K=4$. This agrees with our analysis above. On the other hand, in most of the randomly generated scenarios, there are only small performance differences between dual-path and triple-path/unlimited-path routing. This in turn supports our decision on limiting the route selection to dual-path routes, which strikes a balance between the network performance and the complexity of system implementation.

The Jain's fairness index of bandwidth allocation in the 10 simulated scenarios are $\{1,1,1,0.9977,1,0.9290,1$, $0.8939,1,0.9999\}$ respectively. We observe that even the worst scenario yields a fairness index of 0.8939 , which proves that the introduction of the fairness component in our objective function can effectively balance the bandwidth allocation and avoid starvation on some of the APs.

\section{Random Topology}

We now consider random-topology networks where 49 mesh nodes are uniformly randomly placed in a $1500 \times 1500$ square meter area. We assume that the network is a planar graph with maximum degree of 4 . Link capacity is only dependent on the link distance. An example random topology is shown in Fig. 8(a), where the number along each link represents the link capacity (in Mbps). Again, three APs and three GNs are randomly selected in each scenario. Simulation results are plotted in Fig. 9 where each point is averaged over 10 scenarios.

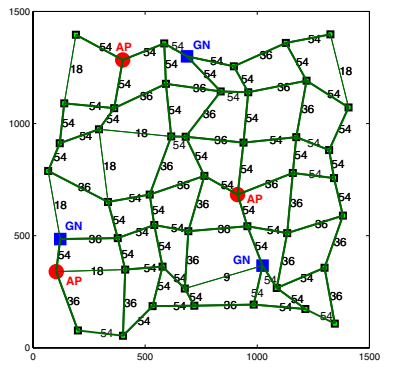

(a) An example network with the number along each link representing the link capacity (in Mbps)

Fig. 8. Random-topology networks with 49 nodes

Compared with that in the grid-topology network, the performance gain of multi-path routing over single-path routing is less significant in random-topology networks. The reason 


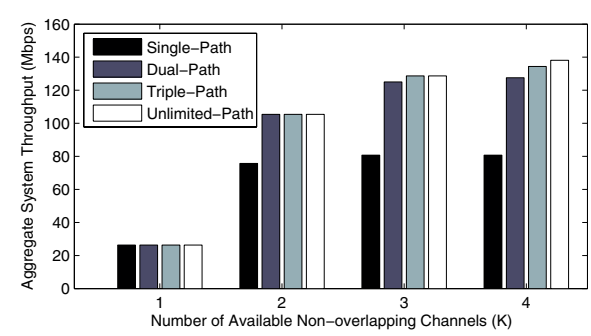

Fig. 9. Comparison of aggregate system throughput in random-topology networks

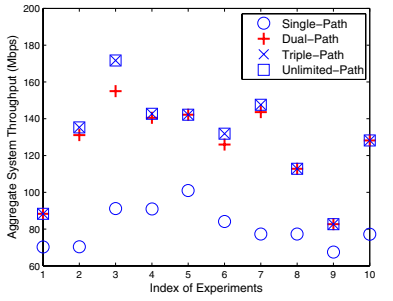

(a) 3 non-overlapping channels

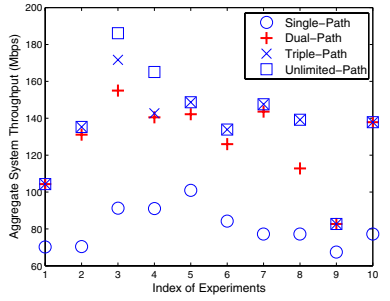

(b) 4 non-overlapping channels
Fig. 10. Results of 10 simulated scenarios in random-topology networks

is that, due to the link capacity diversity in random-topology networks, there may exist bottleneck regions where even multipath routing can not detour around or find better paths.

Another observation in Fig. 9 is that the performance gap between dual-path routing and triple-path/unlimited-path routing is even more narrowed than that in the grid-topology network. One explanation for this phenomenon is that the interference problem becomes more severe as the number of routing paths increases, especially in random-topology networks where two links that are more than two hops away may still interfere with each other. Note that, though we assume that the transmit power of each directional antenna has been adjusted properly, the interference pattern is still difficult to predict when mesh nodes are randomly placed.

In order to show insight of the performance difference of simulated routing protocols in random-topology networks, we plot the simulation results of all the 10 simulated scenarios in Fig. 10. We can see that single-path routing performs differently in 10 scenarios, mainly due to non-uniform link capacities and unpredictable bottlenecks along routing paths. In contrast, triple-path and unlimited-path routing are more likely to achieve higher throughput. Fig. 8(b) shows the routing paths when unlimited-path routing is used in Scenario \#3, which yields significantly higher throughput than dual-path routing because there exist more than two fully or partially disjoint paths from each AP to its associated GN. However, the performance gain of triple-path or unlimited-path routing over dual-path routing is limited on average.

The Jain's fairness index of bandwidth allocation in the 10 simulated scenarios are $\{0.9996,0.9886,0.9953,0.9863$, $0.9957,0.9511,0.9327,0.9154,0.9980,0.9703\}$ respectively. Clearly, the bandwidth is almost evenly distributed to all APs in most scenarios despite the link capacity diversity in random- topology networks. These results again convincingly confirm the effectiveness of the proposed scheme which achieves high aggregate system throughput while maintaining fairness among APs, both of them are highly desirable in WMN applications.

\section{CONCLUSION}

In this paper, we study the problems of system throughput maximization and fair service provisioning in multi-radio multi-channel WMNs with directional antennas. We propose a novel algorithm to produce joint decisions on routing and channel assignment with practical implementation considerations. Since this problem is known to be NP-hard, we formulate it as a Mixed Integer Programming problem and solve it using the CPLEX optimizer. Through extensive simulation study, we show that our scheme (i.e., the solution to the MIP problem) efficiently exploit the multi-radio multi-channel network architecture and directional antennas in WMNs, which lead to drastically improved aggregate system throughput while maintaining fair bandwidth allocations among APs. Moreover, we translate practical implementation considerations into feasibility constraints in the MIP problem; as a result, our scheme is simple and easy to implement, thus facilitating its deployment with commercial wireless networking devices such as IEEE 802.11 compliant devices.

\section{REFERENCES}

[1] I. Akyildiz, X. Wang, and W. Wang, "Wireless mesh networks: a survey," in Elsevier Computer Networks Journal, 2005.

[2] IEEE 802.11a, Part 11: Wireless LAN Medium Access Control (MAC) and Physical Layer (PHY) Specifications: High-speed Physical Layer in the $5 \mathrm{GHz}$ Band, Supplement to IEEE 802.11 Standard, Aug. 1999.

[3] IEEE 802.11, Part 11: Wireless LAN Medium Access Control (MAC) and Physical Layer (PHY) Specifications, Aug. 1999.

[4] QualNet Simulator, http://www.scalable-networks.com/, Online Link.

[5] J. Tang, G. Xue, and W. Zhan, "Maximum throughput and fair bandwidth. allocation in multi-channel wireless mesh networks," in Proc. IEEE INFOCOM'06, 2006.

[6] J. Tang, G. Xue, and W. Zhang, "Interference-aware topology control and qos routing in multi-channel wireless mesh networks," in $A C M$ MobiHoc'05, 2005.

[7] A. Raniwala, K. Gopalan, and T.-C. Chiueh, "Centralized channel assignment and routing algorithms for multi-channel wireless mesh networks," in ACM SIGMOBILE Mobile Computing and Communications Review $(M C 2 R), 2004$.

[8] W.-H. Tam and Y.-C. Tseng, "Joint multi-channel link layer and multipath routing design for wireless mesh networks," in Proc. IEEE INFOCOM'07, 2007.

[9] A. Raniwala and T.-C. Chiueh, "Architecture and algorithms for an ieee 802.11-based multi-channel wireless mesh network," in Proc. IEEE INFOCOM'05, 2005.

[10] M. Alicherry, R. Bhatia, and L. Li, "Joint channel assignment and routing for throughput optimization in multi-radio wireless mesh networks," in Proc. ACM MobiCom'05, 2005.

[11] M. Kodialam and T. Nandagopal, "Characterizing the capacity region in multi-radio multi-channel wireless mesh networks," in ACM MobiCom'05, 2005.

[12] S. Bandyopadhyay, S. Roy, T. Ueda, and K. Hasuike, "Multipath routing in ad hoc wireless networks with directional antenna," in PWC'02, 2002.

[13] E. Gabrielyan and R. Hersch, "Reliable multi-path routing schemes for real-time streaming," in ICDT'06, 2006.

[14] ILOG CPLEX, http://www.ilog.com/products/cplex/, Online Link.

[15] R. Jain, D. Chiu, and W. Hawe, "A quantitative measure of fairness and discrimination for resource allocation in shared computer system," in DEC Technical Report, 1984. 